\title{
TREINAMENTO DE FORÇA COM USO DE CORRENTES E POTENCIALIZAÇÃO PÓS-ATIVAÇÃO DO SALTO VERTICAL
}

\author{
GRAD. JÉDERSON NUNES \\ Graduado em Educação Física pela Escola Superior de Educação Física, \\ Universidade Federal de Pelotas (ESEF/UFPel). Professor na \\ Viva Fitness Center (Pelotas - Rio Grande do Sul - Brasil) \\ E-mail: jedersonunes@gmail.com

\section{GRAD. SAMUEL MOURA DA ROSA'} \\ Graduado em Educação Física pela Escola Superior \\ de Educação Física, Universidade Federal de Pelotas (ESEF/UFPel) \\ DR. FABRÍCIO BOSCOLO DEL VECCHIO \\ Doutorado em Ciências do Esporte na Faculdade de Educação Física, \\ Universidade Estadual de Campinas (FEF/UNICAMP). Professor da Escola \\ Superior de Educação Física, Universidade Federal de Pelotas \\ (Pelotas - Rio Grande do Sul - Brasil) \\ E-mail: fabricio_boscolo@uol.com.br
}

\begin{abstract}
RESUMO
A Potencialização Pós-Ativação (PPA) é estratégia para melhora do desempenho com uso de carga fixa (RF), definida no ponto de falha mecânica (PFM). Por outro lado, o uso de correntes (CRT) é estímulo com resistência variável (RV). Assim, objetivou-se avaliar os efeitos do CRT na altura e tempo de voo do salto vertical com contramovimento (CMJ). A partir de estudo de intervenção, 15 sujeitos foram avaliados quanto à carga máxima no agachamento (I RM) e altura CMJ. Após isto, executaram três tipos de intervenções: i) RF com 85\% de IRM; ii) $R V$ com $85 \%$ de I RM posicionado no PFM (RV-TF) e, iii) RV com 85\% de I RM posicionado na maior extensão dos joelhos (RV-TV). Observou-se incremento do CMJ para RV-TF e RV-TV, mas não para RF. Conclui-se que houve PPA com os dois estímulos de CRT.
\end{abstract}

PALAVRAS-CHAVE: Educação física e treinamento; exercício; métodos; treinamento de resistência.

l. $\quad$ In memorian. 
A otimização do desempenho esportivo gera busca por diferentes métodos de treino mais eficientes. Dentre as várias estratégias de melhora aguda do rendimento físico está a potencialização pós-ativação (PPA), a qual foi alvo de detalhada revisão de literatura recente em língua portuguesa. (BATISTA et al., 20 I0). Brevemente, pesquisas sugerem que a capacidade de desenvolver força no músculo esquelético pode ser melhorada temporariamente (FRENCH; KRAEMER; COOKE, 2003), sendo que pelo menos dois mecanismos explicam este fato: a maior fosforilação da miosina reguladora de cadeias leve e excitação neural incrementada. (HAMADA et al., 2000).

Neste contexto, a PPA é estratégia para ativar o sistema muscular a partir de determinado exercício (ou aquecimento prévio) e aumentar o resultado de ação motora subsequente. (MIARKA; DEL VECCHIO; FRANCHINI, 20I I; GILBERT; LEES, 2005; YOUNG; BEHM, 2003). Estudos sugerem possível elevação da altura do salto vertical após a PPA por estímulos isométricos. (REQUENA et al., 201 I; GOSSEN; SALE, 2000) e dinâmicos (MCCANN; FLANAGAN, 20 I 0; WEBER et al., 2008).

A PPA tem sido investigada com emprego de diferentes estratégias, explorando ações concêntricas (MCCANN; FLANAGAN, 20 I0), excêntricas, isométricas (GOSSEN; SALE, 2000) e, inclusive, pliométricas. (BATISTA et al., 2003). No entanto, até o presente momento, todos estudos referentes à PPA fizeram uso de resistência fixa (RF), geralmente entre 70\% e 100\% de IRM, a qual decorre de carga máxima obtida próxima ao ponto de falha mecânica (PFM) nos testes de força dinâmica máxima. (KHAMOUI; JO; BROWN, 201 I; McCANN; FLANAGAN, 20 I 0; KHAMOUl et al., 2009). Assim, é possível que toda amplitude de movimento após o PFM seja cumprida com esforço submáximo e decrescente. (CARPINELLI, 20 I I).

Assumindo-se que o movimento realizado após superação do PFM seja feito com esforços submáximos, em função do uso de carga fixa ao longo do exercício, a literatura tem indicado o uso de estímulos com resistência variável (RV). Neste contexto, um dos meios conhecidos para se proporcionar a RV é o treino de força com uso de correntes (chain resistance training [CRT]. (BAKER; NEWTON, 2009; BERNING; COOKER; BRIGGS, 2008). Nele, correntes são adicionadas para compensar a facilidade de execução no final do movimento e minimizar os efeitos do PFM. Elas são dispostas nas extremidades das barras ou halteres, com a vantagem de proporcionar estímulo variável e progressivo. No agachamento ou supino reto, por exemplo, à medida que a barra se eleva durante a fase concêntrica, mais carga é adicionada, e isto aumenta a carga ao seu final, consequentemente eleva o estímulo muscular. (COKER; BERNING; BRIGGS, 2006). 
Em relação à eficiência do CRT, pelo menos dois estudos indicaram que exercícios realizados com este método tendem a aumentar a percepção subjetiva de esforço durante os treinos, mesmo com atletas treinados em levantamento de peso olímpico. (BERNING; COKER; BRIGGS, 2008; COKER; BERNING; BRIGGS, 2006). E, recentemente, observou-se que esta estratégia também é adequada para o aumento da força no supino reto entre atletas de voleibol e basquetebol. (BURNHAM; RUUD; MCGOWAN, 20।0).

Considerando que o PFM é a porção mais difícil da amplitude de movimento, e a carga de IRM restringe a quilagem que o indivíduo consegue mover para além deste ponto (CARPINELLI, 20 I I), o CRT tem sido empregado para elevação da produção de potência nos exercícios de levantamento básico, como agachamento e supino reto, e de educativos para o levantamento olímpico, como o encaixe. (BERING; COKER; ADAMS, 2004; MCMASTER; CRONIN; MCGUIGAN et al., 20 I0). No entanto, do melhor do conhecimento dos autores, nenhum trabalho explorou o CRT como meio de treino para estimular a PPA. Assim, o objetivo da presente investigação foi avaliar os efeitos agudos do treinamento com uso de correntes no salto vertical com contramovimento.

\section{MATERIAL E MÉTODOS}

\section{TIPO DE ESTUDO E CARACTERIZAÇÃO DAS VARIÁVEIS}

Este é um estudo de intervenção, quase-experimental, randomizado (GRATTON; JONES, 20 I 0), delineado para investigar se estímulos de força com resistência variável podem potencializar o salto vertical. As variáveis dependentes são altura e tempo de voo no salto vertical com contramovimento. A variável independente é a execução de cinco repetições no agachamento, com presença ou ausência do uso de correntes (chain resistance training, CRT).

TAMANHO AMOSTRAL E CASUÍSTICA

O tamanho amostral foi calculado a partir de estudo prévio que investigou efeitos do treinamento de força no salto vertical. (ARABATZI; KELLIS; SAEZ-SAEZ DE VILLARREAL, 20I0). Considerando diferença alvo de 5,8 cm, desvio padrão médio de 5,6 cm, 5\% como nível de significância e poder de $80 \%$, seriam necessários I 5 sujeitos. (WHITLEY; BALL, 2002). Como o estudo conta com delineamento contrabalanceado, recrutaram-se oito indivíduos, os quais foram expostos a três modos de intervenção (um com RI e dois com RV), perfazendo total de 24 unidades intervencionais. 
Todos os indivíduos, com idade entre 18 e 26 anos, tinham pelo menos 12 meses de experiência com treinamento de força, estavam familiarizados com o exercício de agachamento e eram proficientes na realização adequada do salto vertical com contramovimento.

\section{PROCEDIMENTOS DE AVALIAÇÃO}

a) Carga máxima

teste de carga máxima foi realizado no aparelho Smith Machine, marca Physicus ${ }^{\circledR}$, com barra pesando 17,4kg. Após aquecimento geral de cinco minutos, que envolveu exercícios calistênicos, de deslocamentos e movimentação corporal, o aquecimento específico, antes do teste, foi feito no mesmo aparelho, porém com carga leve, e autosselecionada. Realizaram-se quatro tentativas com quatro minutos de intervalo entre elas para estimativa da carga. Durante a execução do teste, não se permitiu a retirada das mãos da barra e os avaliados não puderam movimentar os pés. (BROWN; WEIR, 200 I). Complementarmente durante este procedimento, quando ocorreu interrupção da fase concêntrica (MILETELLO; BEAM; COOPER, 2009), um dos autores (IN) registrou o ponto de falha mecânica (PFM), em graus, com auxílio de goniômetro. Este procedimento, embora tenha sido feito com tecnologia diferente, segue as orientações e padronizações de outros estudos que estudaram o PFM em exercício poliarticulares. (DRINKWATER et al., 2007; ESCAMILLA et al., 200 I).

\section{b) Salto vertical}

Foram realizados saltos verticais com contramovimento nos três dias do experimento, sendo estes em série de três saltos (BROWN; WEIR, 200 I), antes e depois dos testes de carga fixa, variável acima (TV) e abaixo (TF). Os saltos foram precedidos por movimentação corporal auto-organizada pelos sujeitos, mas com supervisão de dois co-autores do trabalho, com vistas à manutenção do estado de prontidão. Foram realizados pequenos saltos, alongamentos leves, com durações inferiores a 20 segundos cada posição corporal, e exercícios calistênicos, como polichinelos.

Os saltos foram realizados sobre tapete de contato (Jump System 1.0®, CEFISE, SP/Brasil), sistema eletrônico guarda reprodutibilidade elevada entre medidas, com coeficiente de correlação intraclasse de 0,98 (MARKOVIC et al., 2004) e confiabilidade adequada frente à plataforma de força, com correlação de 0,996. (KENNY; CAIREALLÁIN; COMYNS, 20I2). Nele, a partir da perda de contato com o solo após o salto, calcula-se o tempo de voo, em milissegundos e, então, é estimado o tamanho do deslocamento vertical, em centímetros. Os cuidados quanto à execução do teste de salto com contramovimento são essenciais, pois alterações no padrão de movimento podem sobre ou subestimar a altura do salto. 
Para isto, foram tomados cuidados quanto à execução, os quais são descritos em Braz et al., (2010).

\section{DETERMINAÇÃO DA CARGA DE TREINO}

No TV a carga foi estimada pela amplitude máxima de cada indivíduo com $85 \%$ de IRM, com 70\% da carga sendo invariável (barra e anilha) e I5\% da carga com uso de correntes, com a resistência de $85 \%$ de I RM disposta na maior extensão dos joelhos, ou seja, com o indivíduo em pé (FIGURA I).

No TF a mesma carga alvo foi inicialmente disposta no ponto de falha mecânica (PFM), com o sujeito agachado; porém, durante a fase concêntrica do exercício, a carga variável (de correntes) aumentou progressivamente até a amplitude máxima, de modo que, ao final do movimento, a quilagem total era superior aos $85 \%$ de I RM do início do movimento (FIGURA 2).

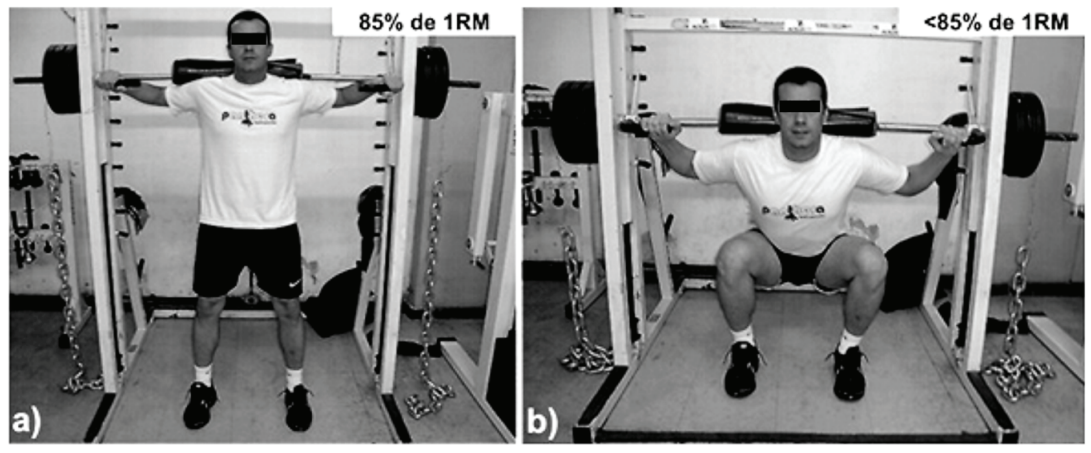

Figura I. Treino com resistência variável com $85 \%$ de IRM colocado na máxima extensão dos joelhos (Treino de Velocidade, TV).
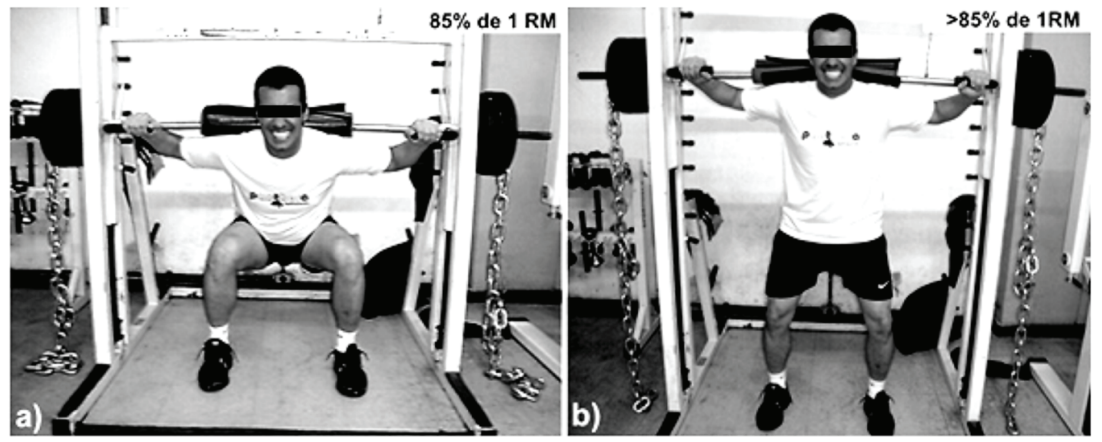

Figura 2. Treino com resistência variável com $85 \%$ de IRM colocado próximo ao ponto de falha mecânica (Treino de Força, TF). 
Para o cálculo da prescrição das cargas, após a identificação do valor da carga de IRM de cada indivíduo, foi considerado:

IRM $=x$, o qual foi colocado na fórmula $85 \%=x \cdot 0,85$, para se encontrar $85 \%$ de IRM na carga fixa. Após identificação desta quilagem, calculou-se $70 \%=$ $\times \cdot 0,7$ para carga fixa e I $5 \%=x \cdot 0,15$ para a carga advinda das correntes.

\section{DELINEAMENTO DA INTERVENÇÃO}

O delineamento do estudo e respectivas intervenções são apresentados na Figura I. No primeiro dia, foram cumpridas cinco etapas diferentes: I) Anamnese e avaliação física, aferindo-se altura, massa e percentual de gordura, calculado de acordo com protocolo de sete dobras. (POLLOCK; WILMORE, 1993). 2) Execução de série de três saltos verticais com contramovimento, com registro: da altura do melhor salto, do tempo de voo do rendimento mediano e do somatório das alturas dos três saltos; após cinco minutos de recuperação, 3) No aparelho Smith Machine, com trena vertical e paralela à barra guiada e ao indivíduo, mediu-se a amplitude de movimento em centímetros, quando o mesmo executou o agachamento profundo no equipamento e foi realizado teste de carga máxima. (BROWN; WEIR, 200 I). 4) Depois de cinco minutos para descanso após o teste de IRM, os indivíduos realizaram a primeira intervenção, considerada como situação controle: série única de cinco repetições com 85\% de IRM, considerado como estímulo de resistência fixa (RF). 5) Passados cinco minutos, considerado tempo de potencialização, os sujeitos realizaram mais três saltos verticais.

Após 48h do término das avaliações e da intervenção com Rl, os indivíduos realizaram os estímulos de treino com carga variável, acrescida do uso de correntes nas extremidades da barra, feitos de forma randomizada, contrabalanceada e seguidos de três saltos verticais cada, a saber:

I) Treino priorizando a velocidade (TV): treino com carga de $85 \%$ de I RM posicionada na altura máxima de extensão dos joelhos de cada indivíduo. Assim, quando a pessoa estivesse no final da fase excêntrica, agachada, a carga seria inferior a 85\% de IRM (resistência acima, FIGURA 2);

II) Treino priorizando a força (TF): a carga de $85 \%$ de IRM foi ajustada para ocorrer quando da realização do agachamento, no ponto de falha mecânica de cada participante (resistência embaixo, FIGURA 3). Desse modo, quando a pessoa atingisse o final da fase concêntrica, a carga seria superior a $85 \%$ de I RM (resistência abaixo, FIGURA 2). 

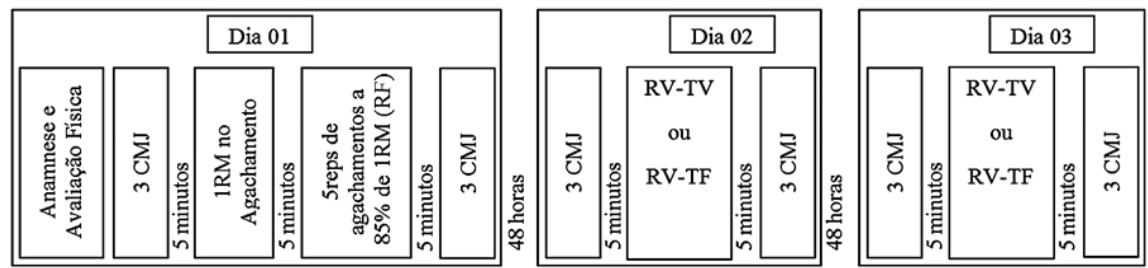

Figura 3. Delineamento do estudo

CMJ: Salto com contramovimento; RM: Repetição máxima; RI: Resistência fixa;

RV: Resistência variável; TV: Treino de velocidade; TF: Treino de força.

Em cada um dos dias de intervenção com carga variável, os participantes executaram duas novas séries de três saltos verticais, intermediadas por uma série de cinco agachamentos e, entre os procedimentos, ocorreram recuperações passivas de cinco minutos (FIGURA 3). Embora a determinação dos estímulos tenha sido feita de modo aleatório, os indivíduos não sabiam qual procedimento seria realizado, de modo a minimizar os aspectos volitivos. Ainda, o estímulo não sorteado na primeira sessão de treino variável ocorreu 48h após o que foi efetuado.

\section{ASPECTOS ÉTICOS DA PESQUISA}

Seguindo as orientações da resolução 196/96 do Conselho Nacional de Saúde, o projeto foi submetido e aprovado pelo Comitê de Ética em Pesquisa com Seres Humanos da Escola Superior de Educação Física da Universidade Federal de Pelotas (Protocolo n $008 / 20$ I I). Após receberem esclarecimentos da proposta do estudo e dos riscos envolvidos, os envolvidos assinaram o Termo de Consentimento Livre e Esclarecido para suas participações.

\section{ANÁLISE DOS DADOS}

Os dados são apresentados como média $\pm d p$. Para as três variáveis consideradas, o desempenho, nos três momentos pré-intervenção, foi testado com a análise de variância de um caminho, e os mesmos foram considerados sem diferenças $(p>0,05)$.

Para verificação da potencialização pós-ativação em cada uma das situações (resistência fixa, acima e abaixo) em separado, foi utilizado o teste $t$ de Student. Para constatação de diferenças no delta de variação percentual $(\Delta \%=100$-[medida pré-intervenção • 100/medida pós-intervenção]), empregou-se a análise de variância de um caminho, e post-hoc de Dunnett (com resistência fixa como situação 
controle). Apesar de $p<0,05$ ser assumido como significante, são apresentados níveis inferiores para indicar a magnitude das diferenças.

\section{RESULTADOS}

As medidas descritivas dos sujeitos da pesquisa são apresentadas na Tabela I . As medidas descritivas para as três variáveis (tempo de voo, altura do melhor salto e soma das alturas de três saltos), nas três intervenções (resistência fixa, resistência acima e resistência abaixo), podem ser encontradas na Tabela 2.

A Tabela 3 mostra a alteração média, em porcentagem, dos três tipos de procedimentos, nas variáveis consideradas. Não houve diferença significativa entre os estímulos com resistência variável acima e resistência variável embaixo, sendo que ambos elevaram a altura de salto (entre 3 e 4,5\%) e tempo de voo. Por outro lado, o delta de variação percentual da resistência fixa foi negativo e diferente das situações com resistência variável $(p<0,05)$, com perda de desempenho de até 3\%.

Tabela I. Medidas descritivas das variáveis antropométricas e relacionadas à I RM da amostra

\begin{tabular}{lc}
\hline Variáveis & média $\pm \mathrm{dp}$ \\
\hline Idade (anos) & $22 \pm 2,93$ \\
Massa corporal $(\mathrm{kg})$ & $73,16 \pm 11,16$ \\
Estatura $(\mathrm{cm})$ & $172 \pm 7$ \\
Gordura corporal $(\%)$ & $8,67 \pm 6,47$ \\
IRM $(\mathrm{kg})$ & $77,38 \pm 11,49$ \\
Deslocamento da barra $(\mathrm{cm})$ & $70,51 \pm 7,17$ \\
Ponto de falha mecânica $\left(^{\circ}\right)$ & $112,5 \pm 6,55$ \\
\hline
\end{tabular}

Com relação à carga invariável, não se observaram diferenças significantes pré e pós-intervenção para as três variáveis analisadas, havendo dados negativos no tamanho do efeito $(T E=-0,24)$, indicando queda de desempenho. Na resistência variável acima (TV), observa-se melhora significativa nas medidas de tempo de voo e altura em um salto, após intervenção $(p<0,05)$. Com relação à intervenção com resistência variável embaixo (TF), também houve diferença significativa no tempo de voo e na altura em um salto (respectivamente $p<0,0$ I e $p<0,00$ I), após a intervenção.

Os valores individuais e a média do grupo (linha tracejada), obtidos pré e pós-intervenção para o tempo de voo, altura de um salto e somatório das alturas de três saltos são apresentados nos gráficos I, 2 e 3, respectivamente. 
Tabela 2. Medidas descritivas do desempenho de salto (tempo de voo e altura), segundo momento e tipo de intervenção

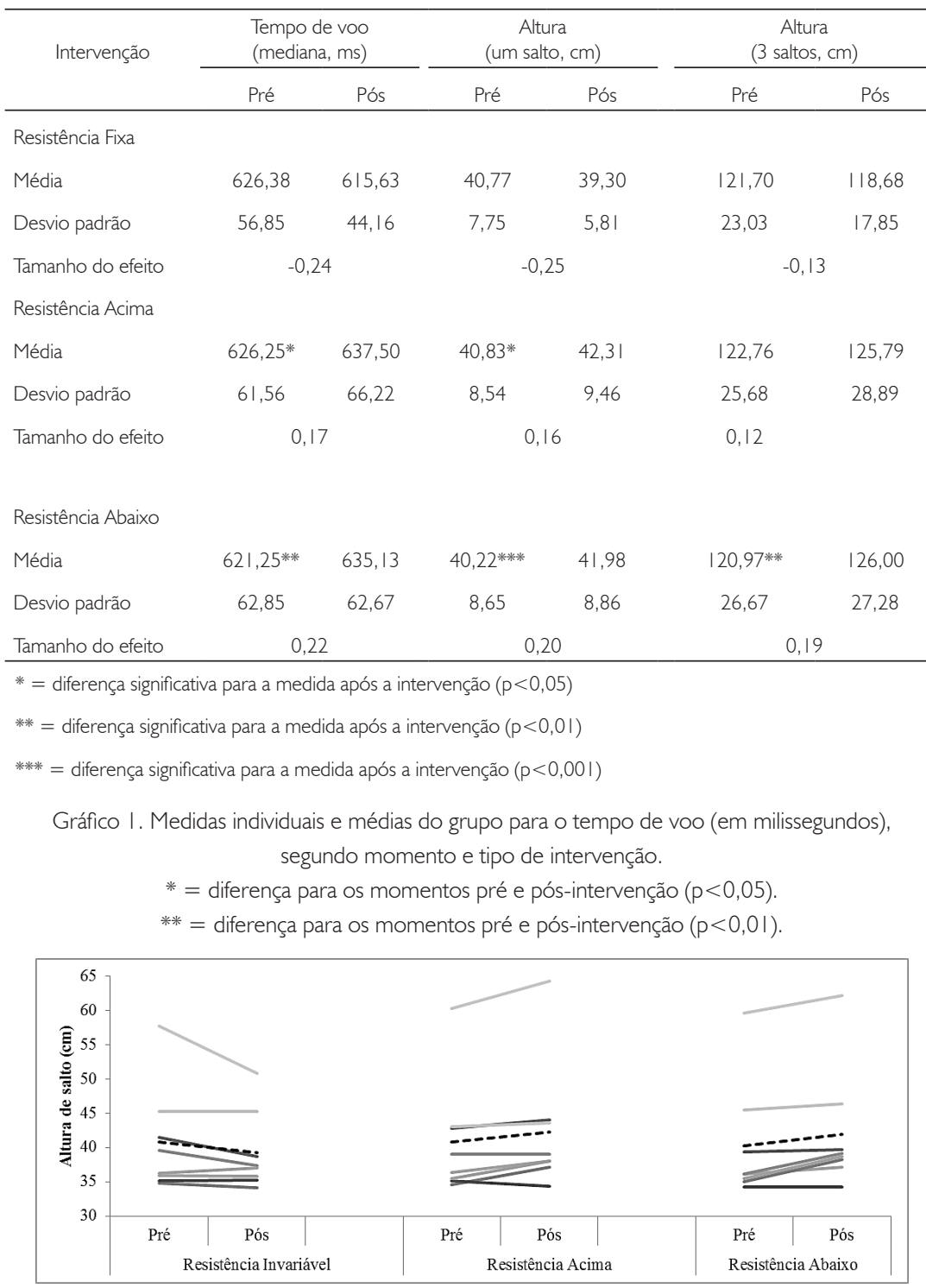

Gráfico 2. Medidas individuais e médias do grupo para a altura média de salto (em centímetros), segundo momento e tipo de intervenção.

* = diferença para os momentos pré e pós-intervenção $(p<0,05)$.

**** $=$ diferença para os momentos pré e pós-intervenção $(p<0,00 \mathrm{I})$. 


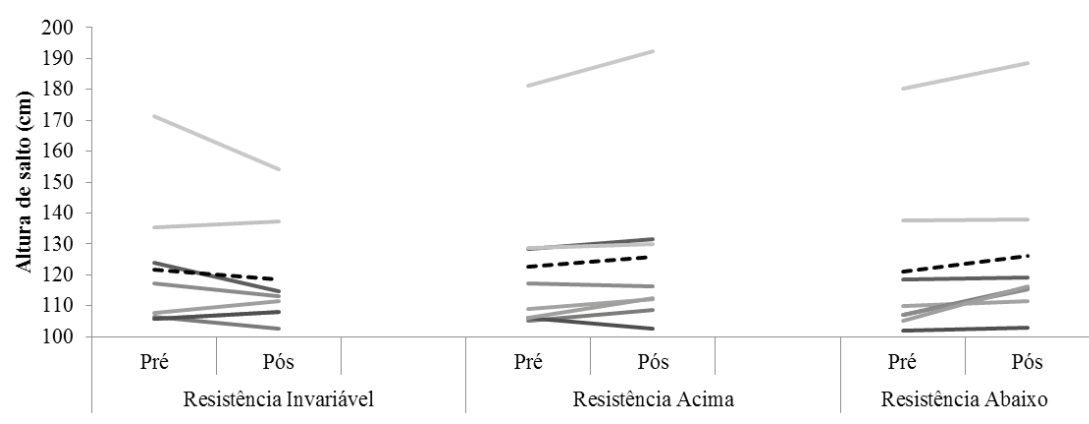

Gráfico 3. Medidas individuais e médias do grupo para a soma de três saltos (em centímetros), segundo momento e tipo de intervenção.

** = diferença para os momentos pré e pós-intervenção $(p<0,0 \mathrm{I})$.

Tabela 3. Delta de variação (média \pm dp em \%) entre os momentos pré e pós-intervenção para os três tipos de procedimento

\begin{tabular}{lccc}
\hline & Tempo de voo (em \%) & Altura para um salto (em \%) & $\begin{array}{c}\text { Altura para } \\
\text { três saltos (em \%) }\end{array}$ \\
\hline Resistência Fixa* & $-1,57 \pm 2,35$ & $-3,02 \pm 4,6$ & $-2,20 \pm 5,31$ \\
Resistência Acima & $1,76 \pm 1,70$ & $3,46 \pm 3,46$ & $2,08 \pm 3,13$ \\
Resistência Abaixo & $2,26 \pm 1,91$ & $4,48 \pm 3,84$ & $3,99 \pm 3,72$ \\
\hline
\end{tabular}

* $=$ diferente das demais intervenções $(p<0,05)$, para todas as variáveis

\section{DISCUSSÃO}

Neste trabalho, que buscou avaliar os efeitos da resistência variável na impulsão vertical, o principal achado foi que o CRT proporcionou PPA, gerando ganhos nas variáveis relacionadas ao salto com contramovimento.

Sabe-se que a produção da potência a partir de cargas pesadas, por volta de $80 \%$ de IRM, decorre da relação entre força e velocidade, da mecânica da contração muscular e associação positiva entre força e potência. Com isto, o treinamento com cargas pesadas resultaria no aumento concorrente na produção da potência máxima. Adicionalmente, o emprego de cargas pesadas está relacionado ao princípio do tamanho para o recrutamento das unidades motoras. (CORMIE; MCGUIGAN; NEWTON, 20I I). Já o uso de cargas leves, até 60\% de I RM, também pode proporcionar incremento na produção de potência em função do aprimoramento da taxa de desenvolvimento da força, execução de movimentos velozes e coordenação intermuscular em velocidades altas. (CORMIE; MCGUIGAN; NEWTON, 20 I I). 
Na presente investigação, o CRT-TF, com carga de 85\% de IRM no ponto de falha mecânica, proporcionou melhoras superiores ao CRT-TV, com a mesma carga atingida na extensão máxima de joelhos, no entanto, sem diferenças entre eles. Especificamente quanto ao agachamento, ausência de diferença entre as estratégias de organização da carga podem ser decorrência da variação na carga ótima para produção de potência neste exercício. Sabe-se que a mesma varia entre 50\% e $80 \%$ de IRM e depende do nível de treinamento, modalidade esportiva e prática pregressa no treinamento resistido. (CORMIE et al., 2007). Assim, é possível que a combinação de cargas leves e pesadas seja o melhor modo de se organizar o treinamento de força com foco no aumento da produção de potência. (CORMIE; MCGUIGAN; NEWTON, 20I I).

A partir do emprego do CRT não há, necessariamente, pontos de falha mecânica ao longo do exercício (GOSS, 20 I0), e ele pode ser realizado com velocidade elevada ao longo de todas as repetições, provavelmente em decorrência da transição mais rápida no ciclo de alongamento encurtamento (CAE) e maior possibilidade de PPA. (BAKER; NEWTON, 2009). Neste contexto, constatou-se desenvolvimento de potência a partir de cargas leves ou pesadas no CRT, quando se partiu da organização da carga com base em $85 \%$ de I RM. Em função deste treino de potência proporcionar estímulo ao CAE, como consequência da potencialização mioelétrica, estimulação do estiramento reflexo e aumento da ativação muscular durante o trabalho concêntrico (RIBEIRO; DEL VECCHIO, 20II), e o mesmo ser relevante para o salto vertical com contramovimento, empregado na presente investigação, observa-se que o CRT pode se constituir como estratégia relevante para a PPA no salto vertical com contramovimento.

Na presente investigação, o uso de resistência fixa não proporcionou PPA para o salto vertical com, inclusive, diminuição de rendimento. Além de outros trabalhos também demonstrarem este fenômeno (KHAMOUI et al., 2009; MCCANN; FLANAGAN, 20I0), pode ser que um único dia para avaliações, teste de I RM e estímulo de resistência fixa tenha afetado o desempenho dos indivíduos e seja considerada uma das limitações deste estudo. Isto se dá, possivelmente, em decorrência da coexistência entre fadiga e a PPA. Ou seja, apesar de a pessoa, por um lado, estar estimulada a realizar a tarefa com maior sucesso, por outro, o estímulo prévio ainda pode estar impactando negativamente no organismo. Neste contexto, com jogadores de rugby, oito minutos de recuperação já foram suficientes para superação da fadiga e observação da PPA de 4,9\% no salto com contramovimento. (KILDUFF et al., 2008).

Por outro lado, elaborou-se o estímulo desta forma (sem randomização), pois a resistência fixa foi admitida como estímulo padrão, ao se observar, na literatura, 
a PPA no salto vertical a partir deste meio de treino no exercício de agachamento. (FRENCH; KRAEMER; COOKE, 2003; GILBERT; LEES, 2005; YOUNG; BEHM, 2003). Adicionalmente, a queda de rendimento poderia ser minimizada ou corrigida com randomização no dia de testes com sobrecarga invariável, dado que só houve randomização nos estímulos de resistência variável acima (TV) e resistência variável abaixo (TF).

Embora pesquisas com uso de correntes sejam iniciais, Baker e Newton (2009) analisaram I 3 jogadores da liga profissional de rugby, sendo que cada sujeito realizou duas séries de três repetições com $75 \%$ de IRM para o supino reto com resistência fixa ou com $60 \%$ de IRM + I5\% com correntes. A condição variável proporcionou diferença próxima a $10 \%$ na velocidade média e de pico em relação ao exercício padrão. Segundo estes autores, o uso de correntes faz com que haja diminuição de sobrecarga na fase excêntrica, sendo que essa diminuição permite que o atleta inicie o movimento na fase concêntrica com maior velocidade, o que explica a PPA com o TF e TV.

Na sobrecarga variável ficou evidenciada potencialização do salto vertical a partir dos estímulos resistência variável acima (TV) e embaixo (TF), o que mostra a viabilidade da prescrição do treinamento com uso de correntes com finalidade de potencializar este gesto motor. No entanto, vale dizer que houve potencialização superior no TF, quando comparado ao TV e, talvez, seja mais adequado o direcionamento da ênfase no estímulo de força antes do ponto de falha mecânica (PFM) do que o foco na velocidade. Provavelmente isto se dá porque, se o músculo maximiza a produção de força por toda amplitude de movimento, então ganhos superiores na força podem ser alcançados. Como no agachamento a maior produção de força muscular ocorre nos últimos 25\% da fase concêntrica, cargas superiores às encontradas no PFM estimularão maior capacidade de PPA com a organização da carga partindo de $85 \%$ de IRM no PFM e elevação da quilagem ao longo do movimento. (BERNING; COKER; ADAMS, 2004). Adicionalmente, o primeiro estudo que envolveu treinamento com uso de correntes por oito semanas com atletas pode notar melhoras decorrentes deste método de treino, devido ao aumento da velocidade da barra no PFM, o que poderia recrutar maior quantidade de fibras do tipo llb e incrementar a produção de força subsequente. (BURNHAM; RUUD; MCGOWAN, 2010).

Estudo prévio observou que o uso do agachamento, com 85\% de IRM, beneficia a execução de série de saltos verticais subsequentes. (WEBER et al., 2008). Cogita-se, neste sentido, que haja maior fosforilação da miosina reguladora de cadeias leves com o estímulo de força do que o estímulo de velocidade (HAMADA et al., 2000), o que proporcionaria maior predisposição muscular ao 
gesto de potência analisado - o salto vertical. Isto se deve ao aumento na velocidade de saída e elevação na aplicação de força nos ângulos finais de extensão dos joelhos, causados pelo aumento progressivo e linear da resistência externa. (MCMASTER; CRONIN; MCGUIGAN et al., 20 I0).

O treino com carga mais pesada (TF, na qual a menor resistência se localizava no PFM) pode ter maior transferência para o salto vertical, dado que resistências mais elevadas são superiores para potencializar gestos nos quais a aplicação de força dure mais de 250 milissegundos. (KAWAMORI; HAFF, 2004). Como o salto vertical tem tempo de aplicação de força entre $980 \pm 160 \mathrm{~ms}$ e $1010 \pm 150 \mathrm{~ms}$ (CORMIE; MCBRIDE; MCCAULLEY, 2009), neste estudo o TF foi superior para potencialização do mesmo.

\section{CONCLUSÕES}

Os resultados verificados nesse estudo permitem concluir que o treino com resistência variável proporcionou potencialização pós-ativação, com destaque para alocação da maior resistência no ponto de falha mecânica. A resistência variável com o uso de correntes parece ser alternativa viável quando se objetiva melhor rendimento em exercício específico de potência muscular que empregue o ciclo de alongamento-encurtamento, como o salto vertical com contramovimento. Claramente, necessita-se que mais estudos sejam realizados sobre este tema.

\section{Chain resistance training and vertical jump post-activation potentiation}

ABSTRACT: The Post-Activation Potentiation (PAP) is a strategy to improve performance, using a fixed load (RF), defined at the sticking point (PFM). Moreover, the use of chains (CRT) is an effort with variable resistance (RV). Thus, the objective was to evaluate the effects of CRT in the countermovement jump (CMJ). With an intervention study, 15 subjects were evaluated for the maximum squat load (IRM) and height in CMJ. After that, they performed three types of interventions: i) RF with $85 \%$ of I RM; ii) RV with $85 \%$ of I RM positioned in PFM (RV-TF), and iii) RV at $85 \%$ of IRM positioned at greater knee extension (RV-TV). It was observed increase in CMJ to RV-TF and RV-TV, but not to RF. It is concluded that there was PPA with the two RV stimulus.

KEY-WORDS: Physical education and training; exercise; methods; resistance training.

\section{Entrenamiento de fuerza con cadenas y la potenciación} post-activación del salto vertical

RESUMEN: La potenciación post-activación (PAP) es estrategia para mejorar el rendimiento, utilizando una carga fija (RF), que se define en el punto de falla mecánica (PFM). Además, el 
uso de cadenas (CRT) es estimulo con resistencia variable (RV). Así, el objetivo fue evaluar los efectos de la CRT en el salto vertical con contramovimiento (CM)). Con estudio de intervención, I 5 sujetos fueron evaluados acerca de la carga máxima en la sentadilla (IRM) y altura en el CMJ. Después de eso, se realizaron tres tipos de intervenciones: i) RF con el 85\% de IRM; ii) RV con el 85\% de I RM en el PFM (RV-TF), y iii) la RV en I RM 85\% situado en la mayor extensión de las rodillas (RV-TV). Se observó aumento de lo CMJ para RV-TF y RV-TV, pero no para la RF. Se concluye que existe PPA con los dos estímulos de RV.

PALABRAS CLAVE: Educación y entrenamiento físico; ejercicio; métodos; entrenamiento de resistencia.

\section{REFERÊNCIAS}

ARABATZI, F.; KELLIS, E.; SAEZ-SAEZ DE VILLARREAL, E. Vertical jump biomechanics after plyometric, weight lifting, and combined (weight lifting + plyometric) training. Journal of Strength and Conditioning Research, Colorado Springs, v. 24, n. 9, p. 2440-2448, aug. 2010.

BAKER, D. G.; NEWTON, R. U. Effect of kinetically altering a repetition via the use of chain resistance on velocity during the bench press. Journal of Strength and Conditioning Research, Colorado Springs, v. 23, n. 7, p. 1941-1946, oct. 2009.

BATISTA, M. A. B. et al. Potencialização: a influência da contração muscular prévia no desempenho da força rápida. Revista Brasileira de Ciência e Movimento, Brasília, v. I I, n. 2, p. 7-12, jun. 2003.

BATISTA, M. A. B. et al. Potencialização pós-ativação: possíveis mecanismos fisiológicos e sua aplicação no aquecimento de atletas de modalidades de potência. Revista da Educação Física/UEM, Maringá, v. 21, n. I, p. 161-174, jan./mar. 2010.

BERNING, J. M.; COKER, C. A.; ADAMS, K. J. Using chain for strength and conditioning. Strength and Conditioning Research, Colorado Springs, v. 26, n. 5, p. 80-84, may 2004.

BERNING, J. M.; COKER, C. A.; BRIGGS, D. The biomechanical and perceptual influence of chain resistance on the performance of the olympic clean. Journal of Strength and Conditioning Research, Colorado Springs, v. 22, n. 2, p. 390-395, mar. 2008.

BRAZ, T. V. et al. Comparação entre diferentes métodos de medida do salto vertical com contramovimento. Revista Brasileira de Ciência e Movimento, Brasília, v. 18, n. 2, p. 43-49, abr.jun. 2010.

BROWN, L.; WEIR, J. ASEP procedures recommendation I: accurate assessment of muscular strength and power. Journal of Exercise Physiology, Arkansas, v. 4, n. 3, p. I-2I, aug. 2001.

BURNHAM, T. R.; RUUD, J. D.; MCGOWAN, R. Bench press training program with attached chains for female volleyball and basketball athletes. Perceptual and Motor Skills, Missoula, v. I I0, n. I, p. 61-68, jan./feb. 2010. 
CARPINELLI, R. N. Assessment of one repetition maximum ( IRM) and I RM prediction equations: are they really necessary? Medicina Sportiva, Rome, v. I5, n. 2, p. 91-102, may. 201।.

COKER, C. A.; BERNING, J. M.; BRIGGS, D. L. A preliminary investigation of the biomechanical and perceptual influence of chain resistance on the performance of the snatch. Journal of Strength and Conditioning Research, Colorado Springs, v. 20, n. 4, p. 887-89I, nov. 2006.

CORMIE, P.; McGUIGAN, M. R.; NEWTON, R. U. Developing maximal neuromuscular power: training considerations for improving maximal power production. Sports Medicine, Auckland, v. 4I, n. 2, p. 125-146, feb. 201।.

CORMIE, P.; MCBRIDE, J. M.; MCCAULLEY, G. O. Power-time, force-time, and velocity-time curve analysis of the CMJ: impact of training. Journal of Strength and Conditioning Research, Colorado Springs, v. 23, n. I, p. 177-186, jan. 2009.

CORMIE, P. et al. Optimal loading for maximal power output during lower-body resistance exercises. Medicine and Science in Sports and Exercise, Hagerstown, v. 39, n. 2, p. 340-349, feb. 2007.

DRINKWATER, E. J. et al. Validation of an optical encoder during free weight resistance movements and analysis of bench press sticking point power during fatigue. Journal of Strength and Conditioning Research, Colorado Springs, v. 21, n. 2, p. 510-517, may 2007.

ESCAMILLA, R. F. et al. A three-dimensional biomechanical analysis of the squat during varying stance widths. Medicine and Science in Sports and Exercise, Hagerstown, v. 33, n. 6, p. 984-998, jun. 2001.

FRENCH, D. N.; KRAEMER, W. J.; COOKE, C. B. Changes in dynamic exercise performance following a sequence of preconditioning isometric muscle actions. Journal of Strength and Conditioning Research, Colorado Springs, v. 17, n. 3, p. 678-685, mar. 2003.

FRIEDMANN-BETTE, B. et al. Effects of strength training with eccentric overload on muscle adaptation in male athletes. European Journal of Applied Physiology, Heidelberg, v. I08, n. 4, p. $821-836$, mar. 2010.

GILBERT, G.; LEES, A. Changes in the force development characteristics of muscle following repeated maximum force and power exercise. Ergonomics, Liverpool, v. 48, n. 6, p. 1576 I 584, sept. 2005.

GOSS, K. Lifting chains revisted: bigger faster stronger, Utah, v. 4, n. 4, p. 40-42, jul./aug. 2010. Acesso em: 19 fev. 2012. Disponível em: <http://www.biggerfasterstronger.com/ home/MagDetails.asp?id=1734\&previd $=41>$.

GOSSEN, E. R.; SALE, D. G. Effect of postactivation potentiation on dynamic knee extension performance. European Journal of Applied Physiology, Heidelberg, v. 83, n. 6, p. 524-530, dec. 2000. 
GRATTON, C.; JONES, I. Research methods for sports studies. $2^{\text {nd }}$ ed. London: Routledge, Sept 2010.

HAMADA, T. et al. Postactivation potentiation, fiber type, and twitch contraction time in human knee extensor muscles. Journal of Applied Physiology, Bethesda, v. 88, n. 3, p. 21 3 I2137 , jun. 2000.

KHAMOUI, A. V. et al. Effect of potentiating exercise volume on vertical jump parameters in recreationally-trained men. Journal of Strength and Conditioning Research, Philadelphia, Colorado Springs, v. 23, n. 5, p. 1465-1469, aug. 2009.

KHAMOUI, A. V.; JO, E.; BROWN, L. Postactivation potentiation and athletic performance. NSCA hot topic series: Philadelphia. 20I I. Disponível em: <www.nsca-lift.org/HotTopic/ download/Postactivation\%20Potentiation.pdf>. Acesso em: 19 fev. 2012.

KAWAMORI, N.; HAFF, G. The optimal training load for the development of muscular power. Journal of Strength and Conditioning Research, Colorado Springs, v. I8, n. 3, p. 675684, aug. 2004.

KENNY I. C.; CAIREALLÁIN, A. O.; COMYNS, T. M. Validation of an electronic jump mat to assess stretch shortening cycle function. Journal of Strength and Conditioning Research, Colorado Springs, v. 26, n. 6, p. 1601-1608, 2012.

KILDUFF, L. P. et al. Influence of recovery time on post-activation potentiation in professional rugby players. Journal of Sports Sciences, London, v. 26, n. 8, p. 795-702, jun. 2008.

MARKOVIC, G. et al. Reliability and factorial validity of squat and countermovement jump tests. Journal of Strength and Conditioning Research, Colorado Springs, v. I8, n. 3, p. 55।555, mar. 2004.

MCMASTER, D. T.; CRONIN, J.; MCGUIGAN, M. R. Quantification of rubber and chainbased resistance modes. Journal of Strength and Conditioning Research, Colorado Springs, v. 24, n. 8, p. 2056-2064, aug. 2010.

MCCANN, M. R.; FLANAGAN, S. P. The effects of exercise selection and rest interval on postactivation potentiation of vertical jump performance. Journal of Strength and Conditioning Research, Colorado Springs, v.24, n.5, p. 1285-1291, may 2010.

MIARKA, B.; DEL VECCHIO, F. B.; FRANCHINI, E. Acute effects and postativation potentiation in the Special Judo Fitness Test. Journal of Strength and Conditioning Research, Colorado Springs, v. 25, n. 2, p. 427-431, feb. 201 I.

MILETELLO, W. M.; BEAM, J. R.; COOPER, Z. C. A biomechanical analysis of the squat between competitive collegiate, competitive high school, and novice powerlifters. Journal of Strength and Conditioning Research, Colorado Springs, v. 23, n. 5, p. 161 1-1617, may 2009.

POLLOCK, M. L.; WILMORE, J. H. Exercícios na saúde e na doença. 2. ed. Rio de Janeiro: Medsi, 1993. 
REQUENA, B. et al. Relationship between postactivation potentiation of knee extensor muscles, sprinting and vertical jumping performance in professional soccer players. Journal of Strength and Conditioning Research, Colorado Springs, v. 2 I , n. 2, p. 2, p. 367-373, feb. 201 I .

RIBEIRO, Y. S.; DEL VECCHIO, F. B. Metanálise dos efeitos agudos do alongamento na realização de corridas curtas de alta intensidade. Revista Brasileira de Educação Física e Esporte, São Paulo, v. 25, n. 4, p. 567-81, out./dez. 201 I

WEBER, K. et al. Acute effects of heavy-load squats on consecutive squat jump performance. Journal of Strength and Conditioning Research, Colorado Springs, v. 22, n. 3, p. 726-730, may 2008.

WHITLEY E.; BALL J. Statistics review 4: sample size calculations. Critical Care, Bristol, v. 6, n. 3, p. 335-34I, may 2002.

YOUNG, W. B.; BEHM, D. G. Effects of running, static stretching and practice jumps on explosive force production and jumping performance. Journal of Sports Medicine and Physical Fitness, Ballarat, v. 43, n. I, p. 21 -27, mar. 2003.

Recebido: 08 dez. 2010

Aprovado: 5 mar. 2012

Endereço para Correspondência:

Fabrício Boscolo Del Vecchio

Rua Luiz de Camões, 625.

Cohab Tablada,

Pelotas-RS.

CEP: 96055-630. 